\title{
Overexpression of Ku80 correlates with aggressive clinicopathological features and adverse prognosis in esophageal squamous cell carcinoma
}

\author{
SHUAI WANG ${ }^{1}, \mathrm{ZHOU}_{\mathrm{WANG}}{ }^{1}, \mathrm{YU}_{\mathrm{YANG}}{ }^{2}, \mathrm{MO} \mathrm{SHI}^{1}$ and $\mathrm{ZHENGUO} \mathrm{SUN}^{1}$ \\ Departments of ${ }^{1}$ Thoracic Surgery and ${ }^{2}$ Pathology, Provincial Hospital Affiliated to Shandong University, \\ Jinan, Shandong 250021, P.R. China
}

Received September 28, 2014; Accepted May 22, 2015

DOI: $10.3892 / 01.2015 .3635$

\begin{abstract}
Ku80, a subunit of the heterodymeric $\mathrm{Ku}$ protein, is clearly implicated in nonhomologous end joining DNA repair, chemoresistance and radioresistance in malignant tumors. In the present study, the clinicopathological significance of Ku80 in esophageal squamous cell carcinoma (ESCC) was investigated. The expression levels of Ku80 were determined by reverse transcription-quantitative polymerase chain reaction and immunohistochemistry in ESCC specimens and normal esophageal mucosa. The mRNA and protein levels of Ku80 were significantly higher in ESCC tissues than in normal esophageal mucosa, and were significantly associated with tumor differentiation, local invasion, lymph node metastasis and tumor-node-metastasis (TNM) stage. However, overexpression of Ku80 mRNA and protein levels were not significantly correlated with age, gender, tumor site or tumor size. Cox proportional hazards regression model demonstrated that tumor local invasion, lymph node metastasis, TNM stage and Ku80 mRNA and protein levels were independent risk factors indicating the overall survival of patients with ESCC. The present study demonstrated that aberrant Ku80 overexpression is observed in ESCC. In addition, high expression levels of Ku80 are associated with adverse clinicopathological features and unfavorable prognosis in ESCC patients.
\end{abstract}

\section{Introduction}

Esophageal squamous cell carcinoma (ESCC) is a malignant disease with uneven geographical distribution worldwide. Notably, the age of patients at the time of diagnosis is

Correspondence to: Professor Zhou Wang, Department of Thoracic Surgery, Provincial Hospital Affiliated to Shandong University, 324 Jingwu Road, Jinan, Shandong 250021, P.R. China E-mail: wz620226@hotmail.com

Key words: esophageal squamous cell carcinoma, Ku80, prognosis, pathology, clinical relevance reducing (1). In East Asia (China, Japan and South Korea), ESCC has high incidence and mortality rates, and accounts for $90 \%$ of all cases of esophageal carcinoma (2). Despite advances in combined modality therapies for ESCC, including surgery, chemotherapy and radiotherapy, the prognosis for patients remains poor, and the 5-year overall survival rate is $20-30 \%$ (3). Although a number of genetic and epigenetic alterations associated with ESCC have been described (for example, p53, Bcl-2, epidermal growth factor receptor, RUNX3 and esophageal cancer related gene 1) (4-6), its oncogenesis and pathogenesis remain largely unknown. Optimal and commonly-used molecular markers to facilitate the comprehensive management of patients, including early diagnosis and prognostic evaluation, have not been proposed thus far. The prognosis of ESCC patients is tumor-node-metastasis (TNM) stage-specific, but the TNM stage is not sufficiently sensitive to evaluate the prognosis of patients (7). Therefore, the discovery of biomarkers for therapeutic development and prognostic prediction is required.

ESCC is characterized by genome instability through severe DNA damage caused by several factors, including the consumption of food or beverages at high temperatures, tobacco smoking, poor nutrition, infection and heredity (8). $\mathrm{Ku} 80$, one of the subunits of the $\mathrm{Ku} 80 / \mathrm{Ku} 70$ heterodimer, is a central element in the nonhomologous end joining (NHEJ) DNA repair pathway. Double-strand DNA breaks (DSB) activate the catalytic subunit of the DNA-dependent protein kinase and trigger NHEJ repair activities (9). It has been demonstrated that the silencing of Ku80 proteins prevents DSB repair and telomere maintenance, and results in cell cycle arrest, apoptosis, chemosensitivity and radiosensitivity $(10,11)$. Previous studies have demonstrated that $\mathrm{Ku} 80$ is associated with pathological processes in certain malignant tumors, and have provided valuable information regarding the expression levels of $\mathrm{Ku} 80$ and its pathological role in ESCC (11-14). Yang et al (11) reported that Ku80 participates in tumorigenesis, radioresistance and chemoresistance in esophageal cancer cells. Tonotsuka et al (14) observed that Ku80 protein was expressed in the nuclei of basal cell layers and luminal cell layers in esophageal cancer tissues. However, the protein expression pattern of Ku80 and its clinicopathological significance in ESCC is not well 
established. In the present study, the expression levels of Ku80 in ESCC tissues were analyzed, and the association of Ku80 expression with the clinicopathological features and prognosis of patients affected with ESCC were further investigated.

\section{Materials and methods}

Ethics statement. The current study protocol was reviewed and approved by the Research Ethics Committee of the Provincial Hospital Affiliated to Shandong University (Jinan, China) (2003-063). All participants provided written informed consent for the detection of Ku80 in the tissue-derived samples, subsequent data analysis and publication of the results.

Patients and samples. From January until May 2003, 119 patients with ESCC (41 females and 78 males; mean age, $57.8 \pm 12.3$ years) were screened in the Provincial Hospital Affiliated to Shandong University. These patients were diagnosed with ESCC by histopathological detection at the Pathology Department of the hospital, precluding esophageal leiomyoma or other benign disease and malignant tumors originated from organs other than the esophagus. Eligibility was granted if primary diagnosis occurred $\leq 6$ months prior to study enrollment and patients had not received chemotherapy, radiotherapy or biotherapy prior to sample collection. Full medical examinations were recorded, and the clinicopathological characteristics of the patients were analyzed. All the ESCC patients included in the present study were restaged according to the 2009 International Union Against Cancer TNM staging guidelines for esophageal cancer (15). The histopathological evaluation of the samples was performed according to the criteria proposed by the World Health Organization (16).

In the control group, 109 volunteers (35 females and 74 males; mean age, 56.6 \pm 13.2 years) from the Provincial Hospital Affiliated to Shandong University were screened as normal subjects without malignant disease. Their medical records indicated the absence of drug, tobacco and alcohol abuse. All individuals were Han Chinese without consanguineous relationships. Detailed questionnaires were completed by the subjects participating in the present study. The questionnaires collected information about the individuals, including medical and family history, use of over-the-counter medications and exposure to dietary carcinogens. The detailed questionnaires were used to measure average dietary intake 1 year prior to the date of selection for the current study. No significant differences were observed between the 119 ESCC patients and the 109 healthy volunteers (regarding age, gender, medical and family history, smoking status, exposure to dietary carcinogens and dietary habit). All patients fasted $\geq 12 \mathrm{~h}$ and had not smoked for 6 months prior to the collection of tissue samples.

In the ESCC group, 119 pairs of samples were collected from the 119 patients by gastroscopy. Each pair of samples consisted of ESCC tissue and corresponding healthy mucosa (CHEM).

The corresponding healthy esophageal mucosa was harvested from a position $>5 \mathrm{~cm}$ in distance from the margin of the ESCC. A total of 109 normal esophageal mucosa (NEM) samples from the control group were harvested via gastroscopy. The macroscopic examination of the healthy mucosa revealed no signs of deterioration and necrosis. Light microscope examination demonstrated that the healthy mucosal tissues were free of tumor and any detectable concurrent disease, including esophagitis and dysplasia. Following encapsulation in tin foil wrapper, the tissue samples were rinsed in cold $\mathrm{NaCl}(0.9 \%)$, and immediately stored at $-80^{\circ} \mathrm{C}$ until further use.

$R N A$ extraction and reverse transcription-quantitative polymerase chain reaction $(R T-q P C R)$. Total RNA was isolated from esophageal mucosal tissues using TRIzol (Invitrogen Life Technologies, Carlsbad, CA, USA), according to the manufacturer's instructions. The RT system included $2 \mu \mathrm{g}$ RNA, oligo-dT15 primer and M-MLV reverse transcriptase (Takara Bio, Inc., Shiga, Japan) in a volume of $20 \mu \mathrm{l}$ diethylpyrocarbonate-treated water (Takara Shuzo Co., Ltd., Kyoto, Japan). Gene-specific primer sequences (Dingguo Changsheng Biotechnology Co., Ltd., Beijing, China) were as follows: Ku80, F 5'-ACGATTTGGTACAGATGGCACT-3' and R 5'-GCTCCTTGAAGACGCACAGTTT-3' (product, 497 bp); GAPDH, F 5'-GAAGGTGAAGGTCGGAGTC-3' and R 5'-GAAGATGGTGATGGGATTTC-3' (product, 300 bp). RT-qPCR was performed in a LightCycler 480 (Roche Diagnostics, Nutley, NJ, USA) under the following conditions: 1 cycle for $3 \mathrm{~min}$ at $94^{\circ} \mathrm{C}$ followed by 30 cycles of $30 \mathrm{sec}$ at $94^{\circ} \mathrm{C}, 30 \mathrm{sec}$ at $57^{\circ} \mathrm{C}$ and $60 \mathrm{sec}$ at $72^{\circ} \mathrm{C}$. The reactions were terminated at $4{ }^{\circ} \mathrm{C}$, following 5 -min elongation at $72^{\circ} \mathrm{C}$. The expression levels of GAPDH were used as an internal control for RNA quantity and quality. The relative quantity of mRNA (RQ) was calculated as the calibrator-normalized ratio using the LightCycler 480 software, version 1.5 (Roche Diagnostics), applying the following formula: $R Q=2^{-\Delta \Delta C t}$, where $\Delta \Delta \mathrm{Ct}=\left(\mathrm{Ct}_{\text {targeted gene }}-\mathrm{Ct}_{\mathrm{GAPDH}}\right)$ targeted sample $-\left(\mathrm{Ct}_{\mathrm{tar}}\right.$ geted gene $\left.-\mathrm{Ct}_{\mathrm{GAPDH}}\right)$ calibration sample. All the experiments were repeated $\geq 3$ times, and included controls without cDNA, primers or thermophilic polymerase. The specificity of the assay was determined by PCR, using all the primer pairs on each cloned template cDNA to exclude cross-reactivity.

Immunohistochemistry (IHC). IHC staining for Ku80 was performed on $4-\mu \mathrm{m}$ tissue sample sections using an UltraVision Quanto detection system (Thermo Fisher Scientific, Inc., Fremont, CA, USA) following the manufacturer's instructions. Briefly, the sections were incubated with antigen retrieval solution ( $\mathrm{pH}$ 7.0; Beijing Solarbio Science \& Technology Co., Ltd., Beijing, China) for $10 \mathrm{~min}$ at $95^{\circ} \mathrm{C}$ followed by incubation with UltraVision hydrogen peroxide block (Thermo Fisher Scientific, Inc.) for $10 \mathrm{~min}$ to block any endogenous peroxidase activity. Next, the sections were incubated with UltraVision protein block (Thermo Fisher Scientific, Inc.) for $5 \mathrm{~min}$ to reduce nonspecific background staining. Following overnight incubation at $4^{\circ} \mathrm{C}$ with a rabbit monoclonal antihuman Ku80 primary antibody (1:500; cat. no. ab80592; Abcam, Cambridge, UK), the specimens were washed with phosphate-buffered saline (PBS), and incubated with the primary antibody amplifier Quanto (Thermo Fisher Scientific, Inc.) for $10 \mathrm{~min}$, followed by 
10-min incubation with horseradish peroxidase polymer Quanto (Thermo Fisher Scientific, Inc.). Next, the sections were washed with PBS and deionized water, incubated with 3,3-diaminobenzidine (Dingguo Changsheng Biotechnology Co., Ltd.) and counterstained with hematoxylin (Beyotime Institute of Biotechnology, Nantong, China). The specificities of the primary antibodies had been previously confirmed, and their use as positive controls in HeLa cells has also been validated, since previous studies have demonstrated that $\mathrm{Ku} 80$ is overexpressed in these cells (17). The control sections were incubated with PBS instead of the primary antibodies and used as negative controls.

The immunohistochemical scoring of Ku80 was performed using a semiquantitative system as previously reported (18). The specimens were examined under a light microscope. In 5 randomly selected fields per section, the number of immunoreactive cells/100 cells was assessed and quantified as a percentage. Next, the average percentage of the 5 fields was used to assess the proportion score in a 6 -category grading system $(0$, negative; $1,1-10 \% ; 2,11-25 \%$; $3,26-50 \% ; 4,51-75 \%$; and $5,>75 \%$ ). The intensity score for staining was estimated using a 4-category grading system $(0$, negative; 1 , weak; 2 , moderate; and 3 , strong). The IHC score was defined as the proportion score $\mathrm{x}$ the intensity score. The scientists were blinded to the patients data, and scored the samples independently, then reached agreement by repeated analysis and discussion.

Receiver operating characteristics (ROC) curve. The cut-off scores for Ku80 mRNA and protein overexpression levels were screened based on the ROC curve. The raw data corresponding to Ku80 mRNA and protein expression levels in the ESCC and the control groups were analyzed by the MedCalc statistical software package, version 13.0.2.0 (MedCalc Software bvba, Ostend, Belgium). The score closest to the point with maximum sensitivity and specificity was selected as the cut-off score leading to the largest number of patients correctly classified with or without overexpression of Ku80 mRNA and protein levels.

Statistical analysis. All statistical analyses were performed using SPSS software, version 17.0 (SPSS Inc., Chicago, IL, USA). Statistical comparisons between the Ku80 mRNA expression levels in the different groups were performed by analysis of variance (ANOVA). The Mann-Whitney U test was used to determine the differences in Ku80 protein expression levels across the groups. Associations between categorical variables were analyzed using the $\chi^{2}$ test. The survival time was calculated from the date of the diagnosis until mortality or the end of the study. The survival curves were calculated by the Kaplan-Meier method. Univariate log-rank test and Cox regression model analysis were performed to identify prognostic factors. A 2 -tailed $\mathrm{P}<0.05$ was considered to indicate a statistically significant difference.

\section{Results}

High expression levels of Ku80 mRNA in ESCC tissues. The mRNA expression levels of Ku80 were examined in 119 pairs of samples from the ESCC group and in 109 samples from the control group. The relative mRNA expression levels of Ku80 in ESCC tissue, CHEM and NEM were 5.348 \pm 1.480 , $3.327 \pm 1.106$ and $3.149 \pm 1.092$, respectively (Fig. 1A). The mRNA levels of Ku80 in ESCC were significantly higher than in CHEM and NEM (ANOVA; P<0.001). However, no clear difference in the mRNA levels of Ku80 between CHEM and NEM was observed $(\mathrm{P}=0.866)$. According to the ROC curve (Fig. 1B), the threshold value of 4.35 was the closest to the point with maximum sensitivity and specificity of 74.8 and $87.2 \%$, respectively; thus, it was selected as the cut-off value. The area under the ROC curve (AUC) was 0.878 [95\% CI, 0.829-0.918]. Therefore, samples with a calibrator-normalized ratio $>4.35$ were identified as high expression levels of Ku80 mRNA, whereas the remaining samples were considered to have low levels. Consequently, patients were divided into 2 groups; high $(n=84,70.6 \%)$ and low $(n=35,29.4 \%)$ mRNA expression groups. However, in the control group there were $20(18.3 \%)$ cases of high expression of Ku80 mRNA and $89(81.7 \%)$ cases of low expression. Overall, the frequency of high Ku80 mRNA expression levels was significantly increased in ESCC compared with $\operatorname{NEM}\left(\chi^{2}\right.$ test; $\left.\mathrm{P}<0.001\right)$ (data not shown).

High expression levels of Ku80 protein in ESCC tissues. The IHC scores in ESCC tissue, CHEM and NEM were $9.656 \pm 4.633,5.608 \pm 3.759$ and $5.532 \pm 3.741$, respectively (Fig. 1C). The IHC scores of Ku80 in ESCC was significantly higher than those in CHEM and NEM (Mann-Whitney U test; $\mathrm{P}<0.001)$. However, there was no clear difference in IHC scores between CHEM and NEM $(\mathrm{P}=0.268)$. According to the ROC curve analysis (Fig. 1D), the cut-off score was 9, with maximum sensitivity of $63.0 \%$ and specificity of $87.2 \%$. The AUC was 0.756 (95\% CI; 0.695-0.811). Consequently, the patients were divided into 2 groups; high $(n=75,63.0 \%)$ and low $(n=44,37.0 \%)$ protein expression groups. However, there were $18(15.1 \%)$ cases of high expression of Ku80 mRNA and $101(84.9 \%)$ cases of low expression in the control group. The difference in frequency of high protein expression levels between the ESCC tissues and NEM was observed to be statistically significant $\left(\chi^{2}\right.$ test; $\left.\mathrm{P}<0.001\right)$. Spearman bivariate correlation indicated a positive correlation between the mRNA and the protein expression levels of Ku80 ( $r=0.923$; $\mathrm{P}<0.001$ ) (data not shown). Visual analysis of the IHC staining was performed, and positive expression of Ku80 protein was revealed as yellow or brownish yellow stain in the nuclei of tumor cells. Strong positive Ku80 staining was observed in the cancer cell nuclei, whereas negative or weak staining was observed in CHEM and NEM (Fig. 2).

Clinicopathological characteristics of ESCC patients and Ku80 expression levels. The clinicopathological features (age, gender, tumor site, tumor size, differentiation degree, local invasion, lymph node metastasis and TNM stage) of the 119 patients with ESCC in the current study are summarized in Table I. $\chi^{2}$ analysis indicated that the Ku 80 mRNA expression levels positively correlated with differentiation degree $(\mathrm{P}=0.016)$, local invasion $(\mathrm{P}=0.016)$, lymph node metastasis $(\mathrm{P}=0.002)$ and TNM stage $(\mathrm{P}=0.001)$, but not with age $(\mathrm{P}=0.840)$, gender $(\mathrm{P}=0.980)$, tumor location $(\mathrm{P}=0.351)$ or tumor size $(\mathrm{P}=0.407)$ (Table I). In agreement with the mRNA 
A

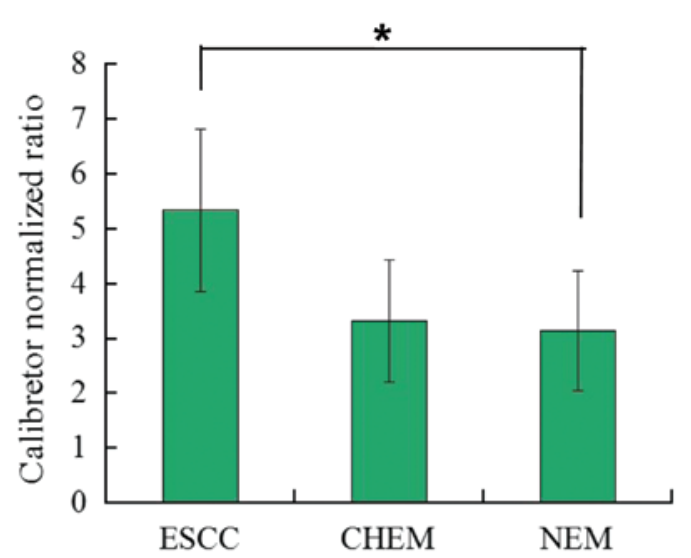

C

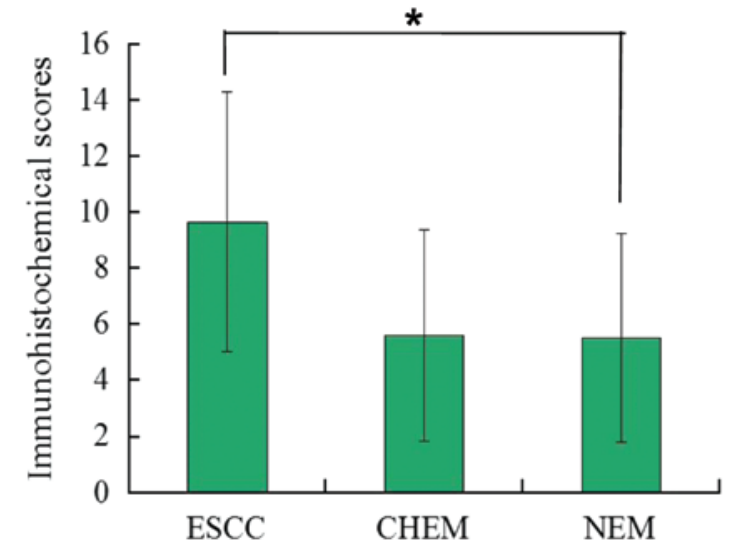

B

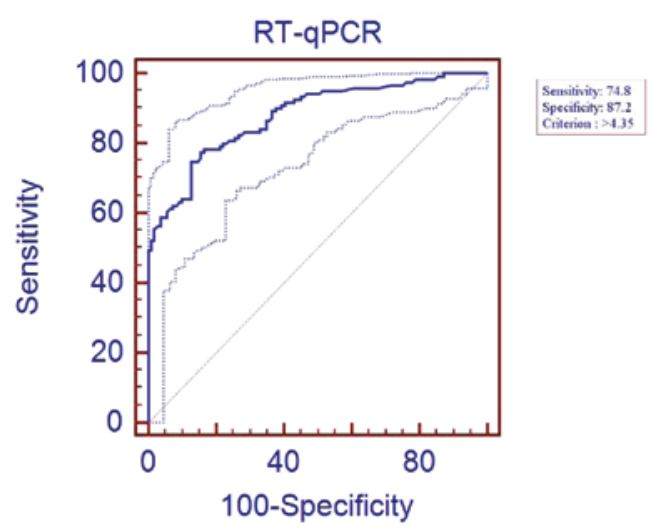

D

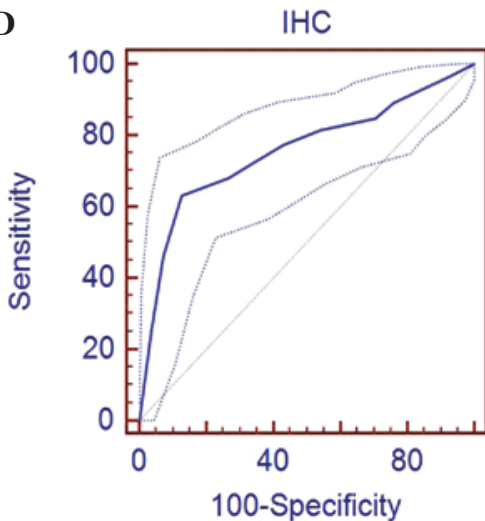

Figure 1. Ku80 is overexpressed in ESCC. (A) RT-qPCR assay of Ku80 mRNA expression levels in 119 cases of ESCC vs. CHEM tissues and 109 cases of NEM. ${ }^{*} \mathrm{P}<0.001$, ESCC vs. NEM. (B) ROC curve analysis for Ku80 mRNA expression levels and selection of the cut-off score. (C) IHC assay of Ku80 protein expression levels in ESCC tissues vs. CHEM and NEM. * $\mathrm{P}<0.001$, ESCC vs. NEM. (D) ROC curve analysis for Ku80 protein expression levels and selection of the cut-off score. The data are presented as the mean \pm standard deviation. ESCC, esophageal squamous cell carcinoma; RT-qPCR, reverse transcriptionquantitative polymerase chain reaction; CHEM, corresponding healthy mucosa; NEM, normal esophageal mucosa; ROC, receiver operating characteristics; IHC, immunohistochemistry.

expression pattern, high expression levels of Ku80 protein presented significant correlations with differentiation degree $(\mathrm{P}=0.001)$, local invasion $(\mathrm{P}=0.004)$, lymph node metastasis $(\mathrm{P}=0.007)$ and TNM stage $(\mathrm{P}=0.002)$, but no correlation was observed between the protein expression levels and age $(\mathrm{P}=0.849)$, gender $(\mathrm{P}=0.842)$, tumor location $(\mathrm{P}=0.906)$ or tumor size $(\mathrm{P}=0.113)$.

Ku80 expression levels are associated with prognosis in patients with ESCC. Patients with ESCC were followed during for a median period of 65.8 months, ranging from 5 to 132 months. The overall 1-, 3-, 5- and 10-year survival rates of the 119 patients were $75.6,54.6,42.0$ and $5.0 \%$, respectively. The median survival time was 46.0 months $(95 \% \mathrm{CI}$, 32.8 59.2 months). As indicated in Table II and Fig. 3, univariate analysis revealed that local invasion $(\mathrm{P}=0.014)$, lymph node metastasis $(\mathrm{P}<0.001)$, TNM stage $(\mathrm{P}<0.001)$ and Ku 80 mRNA and protein levels $(\mathrm{P}<0.001)$ were all significant prognostic factors. However, the age of the patient $(\mathrm{P}=0.406)$, gender $(\mathrm{P}=0.719)$, tumor location $(\mathrm{P}=0.248)$, tumor size $(\mathrm{P}=0.339)$ and differentiation degree $(\mathrm{P}=0.241)$ were not observed to be significant (Table II). Low aggressive local invasion, negative lymph node metastasis, early TNM stage and low Ku80 mRNA and protein expression levels implicated significantly improved prognosis. To exclude confounding factors, the Cox proportional hazards model was used to identify factors associated with overall survival of patients with ESCC. Multivariate analysis revealed that local invasion $(\mathrm{P}=0.011)$, lymph node metastasis $(\mathrm{P}=0.009)$, TNM stage $(\mathrm{P}<0.001), \mathrm{Ku} 80$ mRNA and protein levels $(\mathrm{P}=0.024$ and 0.007 , respectively) were independent significant prognostic factors.

\section{Discussion}

Numerous literature reports have demonstrated genomic and proteomic changes in ESCC (4-7). However, the processes underlying the carcinogenesis and progression of ESCC remain unclear. A previous study indicated that genome instability through severe DNA damage is associated with the carcinogenesis and development of ESCC (8). Ku80 is involved in telomere maintenance, maintenance of chromosomal integrity, recombination of the $\mathrm{V}, \mathrm{D}$ and $\mathbf{J}$ segments during immunoglobulin production, regulation of glucose-regulated peptide 78 gene transcription and cell survival (19-22). Additionally, a number of notable findings regarding the involvement of the Ku80 gene in cancer have been reported previously: The overexpression of Ku80 is clearly associated with the carcinogenesis and the progression of several types 
Table I. Associations between Ku80 expression levels and multiple clinicopathological parameters in ESCC.

\begin{tabular}{|c|c|c|c|c|c|c|c|}
\hline \multirow[b]{2}{*}{ Characteristics } & \multirow[b]{2}{*}{ Cases (119) } & \multicolumn{3}{|c|}{ Ku80 mRNA levels } & \multicolumn{3}{|c|}{ Ku80 protein levels } \\
\hline & & Low (35) & High (84) & P-value & Low (44) & High (75) & P-value \\
\hline Age (years) & & & & 0.840 & & & 0.849 \\
\hline$\geq 60$ & 54 & 15 & 39 & & 19 & 35 & \\
\hline$<60$ & 65 & 20 & 45 & & 25 & 40 & \\
\hline Gender & & & & 0.980 & & & 0.842 \\
\hline Male & 78 & 23 & 55 & & 28 & 50 & \\
\hline Female & 41 & 12 & 29 & & 16 & 25 & \\
\hline Tumor location & & & & 0.351 & & & 0.906 \\
\hline Upper & 12 & 3 & 9 & & 4 & 8 & \\
\hline Middle & 77 & 26 & 51 & & 28 & 49 & \\
\hline Lower & 30 & 6 & 24 & & 12 & 18 & \\
\hline Tumor size & & & & 0.407 & & & 0.113 \\
\hline$\geq 50 \mathrm{~mm}$ & 41 & 10 & 31 & & 11 & 30 & \\
\hline$<50 \mathrm{~mm}$ & 78 & 25 & 53 & & 33 & 45 & \\
\hline Differentiation degree & & & & 0.016 & & & 0.001 \\
\hline G1 & 29 & 14 & 15 & & 19 & 10 & \\
\hline G2 & 57 & 16 & 41 & & 17 & 40 & \\
\hline G3 & 33 & 5 & 28 & & 8 & 25 & \\
\hline Local invasion & & & & 0.016 & & & 0.004 \\
\hline $\mathrm{T} 1+\mathrm{T} 2$ & 57 & 23 & 34 & & 29 & 28 & \\
\hline $\mathrm{T} 3+\mathrm{T} 4$ & 62 & 12 & 50 & & 15 & 47 & \\
\hline Lymph node metastasis & & & & 0.002 & & & 0.007 \\
\hline Positive & 71 & 13 & 58 & & 19 & 52 & \\
\hline Negative & 48 & 22 & 26 & & 25 & 23 & \\
\hline TNM stage & & & & 0.001 & & & 0.002 \\
\hline $\mathrm{I}+\mathrm{II}$ & 53 & 24 & 29 & & 28 & 25 & \\
\hline III + IV & 66 & 11 & 55 & & 16 & 50 & \\
\hline
\end{tabular}

ESCC, esophageal squamous cell carcinoma; TNM, tumor-node-metastasis.

of invasive cancer, including bladder carcinoma (12), gastric carcinoma (13), colorectal carcinoma (23) and breast carcinoma (24). These results suggest that Ku80 may act as an oncogene in various types of cancer.

In the present study, the role of Ku80 in ESCC was explored. It was demonstrated that the mRNA expression levels of Ku80 were increased in ESCC tissues compared with those in CHEM and NEM (Fig. 1). The RT-qPCR results were confirmed by $\mathrm{IHC}$, which revealed that the immunohistochemical scores of Ku80 protein in ESCC were also higher than in CHEM and NEM. Spearman bivariate correlation analysis indicated that the expression levels of Ku80 mRNA were positively correlated with Ku80 protein expression levels. This is consistent with the results reported by Yang et al (11) and Tonotsuka et al (14), who demonstrated that Ku80 was overexpressed in ESCC tissues and cell lines, and suggested that Ku80 may participate in the development of malignant clinicopathological processes in ESCC.

To further explore the clinicopathological significance and prognostic value of Ku80, the 119 ESCC patients were divided into 2 groups of high and low expression, based on the levels of Ku80 mRNA and protein expression. It is difficult to determine the extent to which Ku80 expression is pathologically and clinically relevant, as previous studies had defined the expression levels of Ku80 using different classification systems (10-12). There was no universal and commonly accepted classification system, and the cut-off score was often set arbitrarily. This may lead to numerous errors in studies aiming to evaluate the association of Ku80 expression levels with the clinicopathological features of ESCC. In the present study, an objective, rapid and reproducible scoring method to set the cut-off score based on a ROC curve analysis was used. The value closest to the point with maximum sensitivity and specificity was selected as the cut-off score. By ROC curve analysis, the largest number of ESCC patients was correctly classified into different groups using statistical significance. Thus, there were 84 and 35 patients with ESCC in the high and low Ku80 mRNA level groups, respectively, and 75 and 44 patients in the high and low Ku80 protein level groups, respectively. $\chi^{2}$ test indicated 
Table II. Univariate and multivariate survival analyses for patients with ESCC.

\begin{tabular}{|c|c|c|c|c|c|}
\hline \multirow[b]{2}{*}{ Variable } & \multicolumn{2}{|c|}{ Univariate analysis } & \multicolumn{3}{|c|}{ Multivariate analysis } \\
\hline & $\chi^{2}$ & P-value & HR & $95 \% \mathrm{CI}$ & P-value \\
\hline \multicolumn{6}{|l|}{ Age (years) } \\
\hline$\geq 60$ vs. $<60$ & 0.689 & 0.406 & - & - & - \\
\hline \multicolumn{6}{|l|}{ Gender } \\
\hline Male vs. female & 0.129 & 0.719 & - & - & - \\
\hline \multicolumn{6}{|l|}{ Tumor location } \\
\hline Upper vs. middle vs. lower & 2.791 & 0.248 & - & - & - \\
\hline \multicolumn{6}{|l|}{ Tumor size (mm) } \\
\hline$\geq 50$ vs. $<50$ & 0.916 & 0.339 & - & - & - \\
\hline \multicolumn{6}{|l|}{ Differentiation degree } \\
\hline G1 vs. G2 vs. G3 & 2.848 & 0.241 & - & - & - \\
\hline \multicolumn{6}{|l|}{ Local invasion } \\
\hline $\mathrm{T} 1+\mathrm{T} 2$ vs. $\mathrm{T} 3+\mathrm{T} 4$ & 6.075 & 0.014 & 1.706 & $1.133-2.568$ & 0.011 \\
\hline \multicolumn{6}{|l|}{ Lymph node metastasis } \\
\hline Positive vs. negative & 29.168 & $<0.001$ & 1.898 & $1.173-3.073$ & 0.009 \\
\hline \multicolumn{6}{|l|}{ TNM stage } \\
\hline I+II vs. III+IV & 26.936 & $<0.001$ & 2.612 & $1.638-4.163$ & $<0.001$ \\
\hline \multicolumn{6}{|l|}{ Ku80 mRNA levels } \\
\hline Low vs. high & 13.166 & $<0.001$ & 1.701 & $1.074-2.694$ & 0.024 \\
\hline \multicolumn{6}{|l|}{ Ku80 protein levels } \\
\hline Low vs. high & 17.169 & $<0.001$ & 1.765 & $1.164-2.676$ & 0.007 \\
\hline
\end{tabular}

ESCC, esophageal squamous cell carcinoma; HR, hazard ratio.

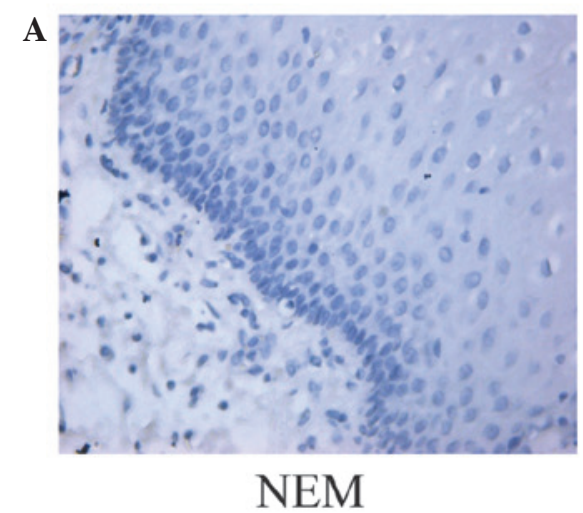

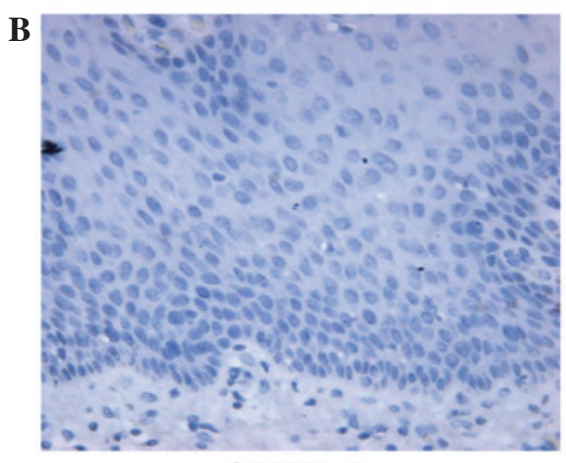

CHEM

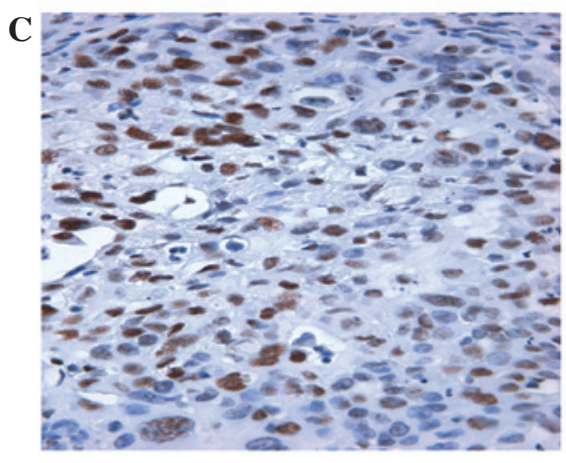

ESCC

Figure 2. Immunohistochemical staining of Ku80 in ESCC specimens. (A) Representative image of negative Ku80 protein expression in NEM at a magnification of $\mathrm{x} 400$. (B) Representative image of negative Ku80 protein expression in CHEM at a magnification of x400. (C) Representative image of negative Ku80 protein expression in ESCC tissues at a magnification of x400 (right panel). NEM, normal esophageal mucosa; CHEM, corresponding healthy mucosa; ESCC, esophageal squamous cell carcinoma.

that the frequency of overexpression was significantly higher in ESCC tissues than in NEM. Additionally, high expression levels of Ku80 were significantly associated with adverse clinicopathological features, including low differentiation degree, aggressive local invasion, lymph node metastasis and advanced TNM stages. Consistent with these results, the data from the IHC analysis indicated that increased Ku80 protein expression levels were associated with low differentiation degree, aggressive local invasion, lymph node metastasis and advanced TNM stages. However, no correlation was observed between the Ku80 mRNA or protein expression levels and several other clinicopathological parameters, including age, gender, tumor location and tumor size, in the current patient cohort (Table I). These findings are consistent with previous reports, which demonstrated that Ku80 expression was associated with important clinicopathological characteristics 

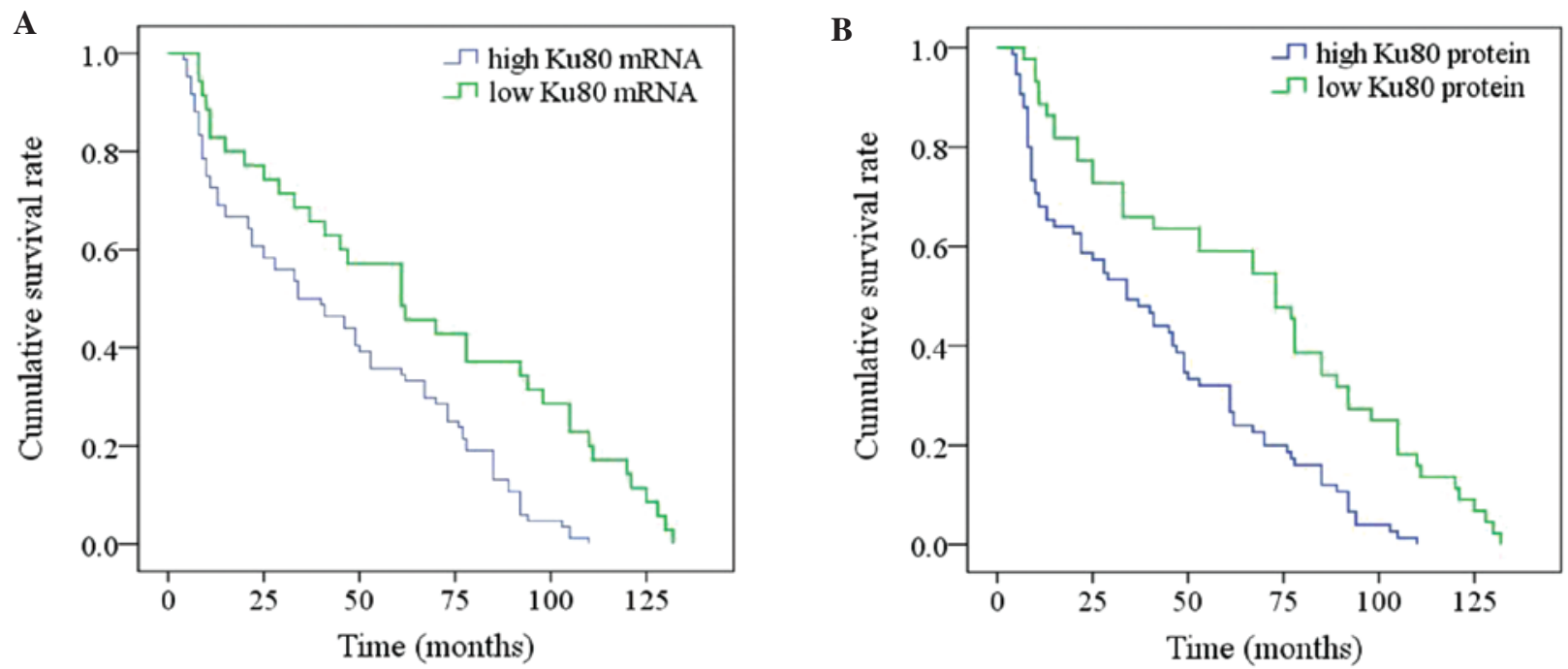

Figure 3. Kaplan-Meier graphs representing the probability of cumulative overall survival in patients with ESCC based on Ku80 expression. High Ku80 (A) mRNA and (B) protein levels were significantly associated with reduced overall survival. ESCC, esophageal squamous cell carcinoma.

in lung adenocarcinoma (25), colorectal cancer (26) and breast cancer (13). These findings suggested that Ku80 was a bona fide oncogene that may be used as a biomarker and a therapeutic target in ESCC.

Previous studies evaluating the prognostic significance of Ku80 expression levels in several types of human cancer reported different conclusions: In lung adenocarcinoma, Ma et al (25) demonstrated an association between Ku80 expression levels and patient outcome in terms of progression-free survival or overall survival. In colorectal cancer, Grabsch et al (26) did not observe any association between high expression levels of Ku80 and long-term survival. In studies of cases of breast cancer, although Alshareeda et al (27) have reported an association with disease-free survival, other authors did not observe such an association (28). These results may reflect the complexity of the DNA damage repair mechanisms in different types of cancer. Therefore, the role of Ku80 in different pathways and networks and its effect on clinical outcome should be analyzed in this complex context. In order to investigate whether Ku80 may be pursued as a novel biomarker for prognostic prediction in patients with ESCC, the prognosis of 119 patients were analyzed in the present study using the Kaplan-Meier method. The current data indicated that the long-term survival of the patients was associated with low aggressive local invasion, negative lymph node metastasis, early TNM stage and low Ku80 mRNA and protein expression levels. Notably, multivariate analysis demonstrated that local invasion, lymph node metastasis, TNM stage and Ku80 mRNA and protein expression levels had independent prognostic influence in patients with ESCC. Therefore, assessment of Ku80 expression in ESCC may provide valuable information regarding the outcome and follow-up management.

Nevertheless, there were various limitations to the present study as follows: Expression of Ku80 in the esophageal mucosa of the individuals investigated may be differentially induced by DNA-damage mutagens. Although numerous potential mutagens, including dietary habits, medicine, alcohol and tobacco were eliminated, the expression levels of Ku80 are affected by environmental mutagens to different extents.
The disease-associated alterations in diet and medication were of small concern, since the patients were diagnosed 6 months prior to the study and did not receive any therapeutic treatment. Human Ku80 is strictly regulated by complex transcriptional and translational mechanisms $(17,21)$. Furthermore, genetic differences among patient cohorts of different geographical origins may result in differences in Ku80 expression. One limitation of the present study is that all participants, who are Han Chinese, come from the same geographical area and have similar genetic background. Adjuvant chemotherapy or radiotherapy may also affect the prognosis of patients. Regarding the treatment modalities, their efficacy was not discussed, since there was no randomized clinical trial. Another limitation of the present study is the limited sample size of the controls used to evaluate the role of Ku80 expression in ESCC. Although, to the best of our knowledge, this is the first report demonstrating that the expression of Ku80 is an independent prognostic factor in patients with ESCC, questions remain unanswered regarding the mechanism of action of Ku80 during the initiation and progression of ESCC. Therefore, further studies with larger samples of cases at the various stages of ESCC are warranted to assess the clinicopathological significance of $\mathrm{Ku} 80$.

In conclusion, the present study provides unique perspectives regarding the involvement of $\mathrm{Ku} 80$ in esophageal carcinogenesis. The data indicate that Ku80 is overexpressed in a significant proportion of cases of ESCC, and it is associated with key clinicopathological features and patient prognosis. In conclusion, the present study provides evidence about the unrecognized roles that Ku80 may perform in tumorigenesis, and its potential use as a novel biomarker and therapeutic target for ESCC.

\section{References}

1. Trivers KF, Sabatino SA and Stewart SL: Trends in esophageal cancer incidence by histology, United States, 1998-2003. Int J Cancer 123: 1422-1428, 2008.

2. Ferlay J, Shin HR, Bray F, Forman D, Mathers C and Parkin DM: Estimates of worldwide burden of cancer in 2008. GLOBOCAN 2008. Int J Cancer 127: 2893-2917, 2010. 
3. Kamangar F, Dores GM and Anderson WF: Patterns of cancer incidence, mortality, and prevalence across five continents: Defining priorities to reduce cancer disparities in different geographic regions of the world. J Clin Oncol 24: 2137-2150, 2006

4. Shibata-Kobayashi S, Yamashita H, Okuma K, Shiraishi K, Igaki $\mathrm{H}$, Ohtomo $\mathrm{K}$ and Nakagawa $\mathrm{K}$ : Correlation among 16 biological factors [p53, p21(waf1), MIB-1 (Ki-67), p16(INK4A), cyclin D1, E-cadherin, Bcl-2, TNF- $\alpha$, NF- $\kappa$ B, TGF- $\beta$, MMP-7, COX-2, EGFR, HER2/neu, ER, and HIF-1 $\alpha$ ] and clinical outcomes following curative chemoradiation therapy in 10 patients with esophageal squamous cell carcinoma. Oncol Lett 5: 903-910, 2013.

5. Wang S, Liu H, Wang Z and Chen HX: Effects of 5-azacytidine on RUNX3 gene expression and the biological behavior of esophageal carcinoma cells. Mol Med Rep 9: 1259-1265, 2014.

6. Rasool S, Khan T, Qazi F and Ganai BA: ECRG1 and its relationship with esophageal cancer: A brief review. Onkologie 36: 213-216, 2013.

7. D'Annoville T, D'Journo XB, Loundou A, Trousse D, Dahan L, Doddoli C, Seitz JF and Thomas PA: Prognostic impact of the extracapsular lymph node involvement on disease-free survival according to the 7th edition of American Joint Committee on Cancer Staging System. Eur J Cardiothorac Surg 44: e207-211, 2013.

8. Kamangar F, Chow WH, Abnet CC and Dawsey SM: Environmental causes of esophageal cancer. Gastroenterol Clin North Am 38: 27-57, 2009.

9. Lieber MR, Gu J,Lu H, Shimazaki N and Tsai AG: Nonhomologous DNA end joining (NHEJ) and chromosomal translocations in humans. Subcell Biochem 50: 279-296, 2010.

10. Fink LS, Lerner CA, Torres PF and Sell C: Ku80 facilitates chromatin binding of the telomere binding protein, TRF2. Cell Cycle 9: 3798-3806, 2010.

11. Yang QS, Gu JL, Du LQ, Jia LL, Qin LL, Wang Y and Fan FY: ShRNA-mediated Ku80 gene silencing inhibits cell proliferation and sensitizes to gamma-radiation and mitomycin C-induced apoptosis in esophageal squamous cell carcinoma lines. J Radiat Res 49: 399-407, 2008.

12. Groselj B, Kerr M and Kiltie AE: Radiosensitisation of bladder cancer cells by panobinostat is modulated by Ku80 expression. Radiother Oncol 108: 429-433, 2013.

13. Kruer TL, Cummins TD, Powell DW and Wittliff JL: Characterization of estrogen response element binding proteins as biomarkers of breast cancer behavior. Clin Biochem 46: 1739-1746, 2013.

14. Tonotsuka N, Hosoi Y, Miyazaki S, Miyata G, Sugawara K, Mori T, Ouchi N, Satomi S, Matsumoto Y, Nakagawa K et al: Heterogeneous expression of DNA-dependent protein kinase in esophageal cancer and normal epithelium. Int J Mol Med 18 : 441-447, 2006.

15. Sobin LH, Gospodarowicz MK and Wittekind C (eds): TNM Classification of Malignant Tumours. 7th edition. Wiley-Blackwell, Oxford, UK, 2009.

16. Hamilton SR and Aaltonen LA (eds): Pathology and genetics of tumours of the digestive system. In: World Health Organization Classification of Tumours. Kleihues P and Sobin LH (eds). IARC Press, Lyon, France, pp237-240, 2000.
17. Koike M, Shiomi T and Koike A: Dimerization and nuclear localization of ku proteins. J Biol Chem 276: 11167-11173, 2001.

18. Song Y, Wang Z, Liu X, Jiang W and Shi M: CCR7 and VEGF-C: Molecular indicator of lymphatic metastatic recurrence in pN0 esophageal squamous cell carcinoma after Ivor-Lewis esophagectomy? Ann Surg Oncol 19: 3606-3612, 2012.

19. Finnie NJ, Gottlieb TM, Blunt T, Jeggo PA and Jackson SP: DNA-dependent protein kinase activity is absent in xrs-6 cells: Implications for site-specific recombination and DNA double-strand break repair. Proc Natl Acad Sci USA 92: 320-324, 1995.

20. Shrivastav M, Miller CA, De Haro LP, Durant ST, Chen BP, Chen DJ and Nickoloff JA: DNA-PKcs and ATM co-regulate DNA double-strand break repair. DNA Repair (Amst) 8: 920-929, 2009

21. Gullo C, Au M, Feng G and Teoh G: The biology of $\mathrm{Ku}$ and its potential oncogenic role in cancer. Biochim Biophys Acta 1765: 223-234, 2006.

22. Liu ES and Lee AS: Common sets of nuclear factors binding to the conserved promoter sequence motif of two coordinately regulated ER protein genes, GRP78 and GRP94. Nucleic Acids Res 19: 5425-5431, 1991.

23. Lim JW, Kim H and Kim KH: Expression of Ku70 and Ku80 mediated by NF-kappa B and cyclooxygenase- 2 is related to proliferation of human gastric cancer cells. J Biol Chem 277: 46093-46100, 2002

24. Hosoi Y, Watanabe T, Nakagawa K, Matsumoto Y, Enomoto A, Morita A, Nagawa $\mathrm{H}$ and Suzuki N: Up-regulation of DNA-dependent protein kinase activity and Sp1 in colorectal cancer. Int J Oncol 25: 461-468, 2004.

25. Ma Q, Li P, Xu M, Yin J, Su Z, Li W and Zhang J: Ku80 is highly expressed in lung adenocarcinoma and promotes cisplatin resistance. J Exp Clin Cancer Res 31: 99, 2012.

26. Grabsch H, Dattani M, Barker L, Maughan N, Maude K, Hansen O, Gabbert HE, Quirke P and Mueller W: Expression of DNA double-strand break repair proteins ATM and BRCA1 predicts survival in colorectal cancer. Clin Cancer Res 12: 1494-1500, 2006.

27. Alshareeda AT, Negm OH, Albarakati N, Green AR, Nolan C, Sultana R, Madhusudan S, Benhasouna A, Tighe P, Ellis IO and Rakha EA: Clinicopathological significance of KU70/KU80, a key DNA damage repair protein in breast cancer. Breast Cancer Res Treat 139: 301-310, 2013.

28. Söderlund Leifler K, Queseth S, Fornander T and Askmalm MS: Low expression of Ku70/80, but high expression of DNAPKcs, predict good response to radiotherapy in early breast cancer. Int J Oncol 37: 1547-1554, 2010. 